\title{
El aprendizaje cooperativo en el desarrollo de habilidades sociales en estudiantes universitarios, Trujillo 2020
}

\author{
José Ricardo Cueva Torres \\ jrcuevat@ucvvirtual.edu.pe \\ Orcid:https://orcid.org/0000-0002-7304-7200 \\ Universidad César Vallejo Trujillo - Perú \\ Dulio Oseda Gago \\ doseda@ucvvirtual.edu.pe \\ Orcid: https://orcid.org/0000-0002-3136-6094 \\ Universidad César Vallejo Trujillo - Perú
}

\section{RESUMEN}

El objetivo de la presente investigación fue determinar el efecto del aprendizaje cooperativo en las habilidades sociales en los estudiantes de la Universidad César Vallejo de Trujillo, 2020. La investigación pertenece al tipo básica, con un diseño no experimental transversal descriptivo correlacional causal. La muestra consistió de 149 estudiantes universitarios. La técnica utilizada fue la encuesta y la muestra fue evaluada a través de dos instrumentos, el primero fue un cuestionario para medir el aprendizaje cooperativo y el segundo para medir las habilidades sociales que fueron validadas con la V de Aiken y evidenciaron ser confiables según el Alfa de Cronbach. Los resultados revelaron que existe relación altamente significativa entre el aprendizaje cooperativo y las habilidades sociales, con un coeficiente de correlación Rho Spearman de $\mathrm{r}=, 645^{* *}$; con significancia de $\mathrm{p}<0.01$. Como conclusión se determinó que existe una vinculación altamente significativa directa entre ambas variables de estudio de los estudiantes universitarios.

Palabras claves: aprendizaje; habilidad y trabajo en equipo. 


\title{
Cooperative learning in the development of social skills in university students, Trujillo 2020
}

\begin{abstract}
The objective of this research was to determine the effect of cooperative learning on social skills in students at Cesar Vallejo University of Trujillo, 2020. The type of this research is basic, with a causal correlational descriptive cross-sectional nonexperimental design. The sample consisted of 149 university students. The technique was the survey, and the sample was measured through two instruments, the first was a questionnaire to measure cooperative learning and the second to measure social skills which were validated with Aiken's V and proved to be reliable according to Cronbach's Alpha. The results revealed that there is a highly significant relationship between cooperative learning and social skills, with a Rho Spearman correlation coefficient of $\mathrm{r}=.645^{* *}$; with a significance of $\mathrm{p}<0.01$. In conclusion, it was determined that there is a highly significant direct relationship between both study variables in the university students.
\end{abstract}

Key words: learning; ability and teamwork.

Artículo recibido: 18. Junio. 2021 Aceptado para publicación: 26. Julio. 2021 Correspondencia:jr_cuevat@hotmail.com Conflictos de Interés: Ninguna que declarar 


\section{INTRODUCCIÓN}

En pleno siglo XXI, se ha ampliado las investigaciones que tratan de exponer claramente las habilidades sociales desde la perspectiva educativa, a pesar que se trata de una antigua aspiración de los docentes, esta concepción se ha enfatizado con más fuerza en los últimos años, a partir de diversas propuestas reflexivas, tomando en consideración la célebre frase, los seres humanos son esencialmente criaturas sociales por antonomasia que conviven y necesitan de los demás para su progreso, realización y supervivencia (Baquero \& Rendón, 2011) El hombre no puede vivir aislado de la sociedad; es un ser en relación con la naturaleza, consigo mismo y con los demás; de modo que "el individuo se hace con los demás, por los demás y para los demás, ya que es en la relación con los demás donde se satisface personalmente" (Rodríguez et al., 2014, p. 154), por ello tiende a organizarse, participar con sus semejantes y trabajar en equipo y, por lo tanto, requiere de habilidades para interrelacionarse con el deseo de alcanzar no sólo el éxito, sino, el bienestar y el saber convivir e interactuar en armonía con los demás.

En este sentido, el sistema educativo peruano, en cuanto al crecimiento de las habilidades sociales podemos afirmar que es precario. Las escuelas en el Perú son instituciones que se han quedado atrapadas en el ayer. Las nuevas generaciones del siglo XXI, no pueden ser educados con modelos pedagógicos y curriculares del siglo XIX y mucho menos, con maestros del siglo XX. (De Zubiria, 2021). Hay que dejar atrás la educación del siglo XIX que aún impera en las escuelas peruanas, ese sistema tradicionalista y reaccionar con seriedad a las exigencias de la sociedad contemporánea, donde los vínculos dependen más del intercambio que de las cargas e imposiciones.

Por otro lado, El mundo laboral exige hoy más que nunca, competencias de tipo colaborativo que contribuya a una mejor gobernanza y mejora de las habilidades sociales. Por lo mismo, es de vital importancia que las organizaciones educativas frente a estos nuevos desafíos trasciendan con nuevas estrategias didácticas innovadoras para desarrollar una educación de calidad. La educación, a todos los niveles, es un espacio importante e indispensable para la formación personal, social y profesional.

En tal sentido, el aprendizaje cooperativo se presenta como una herramienta metodológica que incluye un cambio de mentalidad, actitudes y comportamiento tanto en alumnos como profesor (Sharan, 2014) y desde esta perspectiva, en la realidad 
contextualizada de nuestros estudiantes universitarios se ha observado de manera directa que, generalmente el problema relacionado con las habilidades sociales se produce por la falta del uso de estrategias metodológicas para relacionarse entre ellos, respetar sus potencialidades y limitaciones, demostrar responsabilidad y autonomía, debatir alturadamente intercambiando opiniones y puntos de vista, colaborando con aquellos que más lo necesitan, trayendo como consecuencia que los estudiantes presenten limitaciones para iniciar y mantener conductas que expresan afectividad, comportamientos y voluntades positivas.

Teniendo en cuenta las consideraciones presentadas, el problema de investigación se planteó con la siguiente pregunta: ¿Cuál es el efecto del aprendizaje cooperativo en el desarrollo de las habilidades sociales de los estudiantes de la Universidad César Vallejo de Trujillo?

Esta investigación se justifica por su valor teórico, social, práctico y metodológico. En lo teórico, porque la investigación se fundamenta en teorías contemporáneas que seguirá incrementando el conocimiento científico respecto a estos temas para una posible replicación en algún otro campo de la sociedad. También, tiene una implicancia y relevancia social, por lo trascendente e importante para este tiempo y espacio (Hernández et al., 2014). Hoy en día, el mercado laboral solicita a los individuos nuevas habilidades identificadas con la mejora de las capacidades relacionales como la iniciativa, la colaboración, la asociación y el compromiso. Asimismo, tiene un valor práctico, pues se utilizó instrumentos de aplicación transversal que serán valiosos en la dinámica para conseguir los resultados y objetivos en ambas variables de estudio. Finalmente tiene utilidad metodológica, se utilizó el método científico, la estadística descriptiva y de inferencia, los cuales nos permitieron diseñar y elaborar los dos instrumentos de investigación que pasaran por los criterios de confiabilidad y validación en función de un correcto manejo de información por parte de todos los implicados.

La presente investigación parte del objetivo general: Determinar el efecto del aprendizaje cooperativo en el desarrollo de las habilidades sociales de los estudiantes de la Universidad César Vallejo de Trujillo. Asimismo, se formuló la hipótesis: Existe influencia directa y significativa del aprendizaje cooperativo en el desarrollo de las las habilidades sociales de los estudiantes de la Universidad César Vallejo de Trujillo. 
Respecto a los antecedentes internacionales: Mendo, (2019) en su investigación: "Desarrollo de habilidades sociales y de trabajo en equipo en el contexto universitario: Aprendizaje cooperativo y entrenamiento en habilidades sociales". Su principal conclusión considera que el uso de la metodología cooperativa en el contexto universitario brinda a los estudiantes la oportunidad de fomentar las capacidades relacionales, sociales y de colaboración que pueden ser decisivo en su éxito profesional y social.

Sanchez et al., (2019) en su investigación: "Experiences of cooperative work in higher education. Perceptions about its contribution to the development of social competence". Concluye que el trabajo cooperativo, incluidas las habilidades básicas de relaciones sociales de calidad que solicita la educación europea superior en el círculo personal y profesional, contribuye al progreso y perfeccionamiento de las capacidades sociales, necesidades del mercado social y laboral.

Pegalajar, (2018) en su trabajo de investigación: "Formación en competencias en alumnado universitario de Educación Social mediante prácticas basadas en el aprendizaje cooperativo". La investigación concluye que el aprendizaje cooperativo mejora las habilidades de conversación y colaboración entre los individuos y se ha vuelto valiosa para el avance de las habilidades relacionales entre los discentes de la Universidad de Jaén, España.

Como antecedentes nacional tenemos a Cervantes et al., (2018) en su trabajo de investigación: "Relación profesor - alumno para fomentar el aprendizaje cooperativo de los estudiantes del V ciclo de la USAT”. Su conclusión principal determinó que, existe una relación decente entre instructor - alumno donde se aviva el aprendizaje cooperativo. A medida que el docente sea autoritario y/o permisivo; tanto el quehacer, como los trabajos y las relaciones interpersonales están muy lejos de promover un trabajo cooperativo en las aulas universitarias.

Huambachano y Huaire, (2018) realizaron la investigación: "Desarrollo de habilidades sociales en contextos universitarios" tuvo como objetivo evaluar la efectividad de un programa educativo en la mejora de las habilidades sociales en un grupo de estudiantes de la Universidad Nacional de Educación Enrique Guzmán Y Valle. Los resultados demostraron que mediante metodologías activas y materiales apropiados, trabajar para 
mejorar las habilidades sociales en un determinado momento y contexto, a través de un programa educativo, mejora las prácticas prosociales y la conducta de los alumnos.

Respecto a las bases teórico científicas, el aprendizaje cooperativo se sustentó en la corriente tradicional de (Johnson et al., 1999) y su teoría de la "interdependencia social", conocido también como "El aprendizaje cooperativo en el aula" cuya meta fue, trabajar juntos para obtener resultados favorables incluyendo a todos los integrantes del equipo. Este tipo de aprendizaje se pone en práctica mediante técnicas a partir de la interacción, para que unidos puedan potenciar los conocimientos construidos, así como el de sus compañeros de equipo. En la medida que interactúan los individuos se fortalece esa interdependencia social promoviendo la cooperación que estimula y facilita los esfuerzos para que el otro aprenda.

Para Antón, (2010) el aprendizaje cooperativo es uno de los componentes vitales para que los estudiantes desarrollen su potencial asociado a capacidades mentales superiores. Esto permite a los estudiantes actuar con autonomía, consolidando y garantizando la interacción. Asimismo, este modelo cooperativo ha ido tomando cada vez mayor relevancia debido a las consecuencias que tuvo dentro de los diferentes campos de estudio tales como la educación, la salud, la economía entre otros temas de la vida cotidiana.

Luego por su parte, Caballo, (2007) y su teoría del comportamiento social, que definió a las habilidades sociales como

Conjunto de conductas que permiten al individuo desarrollarse en un contexto individual o interpersonal expresando sentimientos, actitudes, deseos, opiniones o derechos de un modo adecuado a la situación. Generalmente, posibilitan la resolución de problemas inmediatos y la disminución de problemas futuros en la medida que el individuo respeta las conductas de los otros. (p.6)

Como reflejo final de todas las consideraciones presentadas en lo anterior $\mathrm{y}$ motivaciones, encontramos que el aprendizaje cooperativo es, por su propia naturaleza, una manifestación clara de un carácter social e innato en el hombre y con los demás. Creemos que impulsarán y alcanzarán sus objetivos. 


\section{ESTRATEGIAS METODOLÓGICAS O MATERIALES Y MÉTODOS}

\section{Tipo de investigación}

Esta investigación es de tipo básica. Aquí se formula un problema del cual se plantea una hipótesis, sustentada en un marco teórico y sus objetivos respectivos (Hernández et al., 2014). Se construye de manera secuencial y es probatorio.

\section{Diseño de investigación}

De acuerdo a Sánchez et al., (2018), para el estudio de dicha investigación se utilizó un diseño no experimental transversal descriptivo correlacional causal. En los diseños no experimentales no hay manipulación de alguna de las variables; sino que describen, diferencian y examinan asociaciones entre ellas. Es transversal, porque el recojo de datos mediante cuestionarios se hace en un solo instante (en un tiempo rápido y corto); es descriptivo, porque se señala los niveles de variables y dimensiones; es correlacional, porque vinculamos las variables con sus respectivas dimensiones; y es causal, porque por medio de la regresión lineal se determina el porcentaje de influencia de las variables y sus dimensiones (Hernández y Mendoza, 2018).

\section{El respectivo diseño se representa y explica de la siguiente manera:}

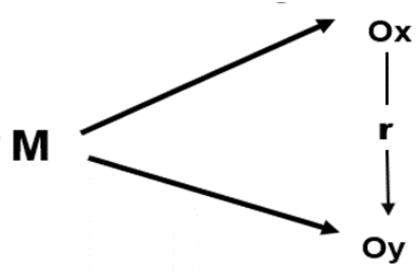

\section{Donde:}

$$
\begin{aligned}
& M=\text { Muestra del estudio (estudiantes universitarios, Trujillo) } \\
& \text { Ox = Observación de la variable relacional } 1 \\
& \text { Oy }=\text { Observación de la variable relacional } 2 \\
& r=\text { Grado de relación entre las variables de estudio. }
\end{aligned}
$$

\section{Población y muestra}

La población estuvo conformada por 243 estudiantes y la muestra por 149 estudiantes de la Universidad César Vallejo. Se hizo uso de la técnica de la encuesta con su instrumento el cuestionario, el cual fue debidamente confiavilizado mediante el Alfa de Cronbach y validado con la V de Aiken 


\section{RESULTADOS Y DISCUSIÓN}

Seguidamente, se muestra los resultados de la variable 1 Aprendizae Cooperativo aplicado a los 149 estudiantes de una universidad privada de Trujillo-2020.

Tabla 1: Categorías de Aprendizaje Cooperativo

\begin{tabular}{cccccc}
\hline & & Frecuencia & Porcentaje & $\begin{array}{c}\text { Porcentaje } \\
\text { válido }\end{array}$ & $\begin{array}{c}\text { Porcentaje } \\
\text { acumulado }\end{array}$ \\
\hline Válido & REG & 2 & 1,3 & 1,3 & 1,3 \\
BUE & 58 & 38,9 & 38,9 & 40,3 \\
MB & 89 & 59,7 & 59,7 & 100,0 \\
Total & 149 & 100,0 & 100,0 & \\
\hline
\end{tabular}

Nota. Data de Aprendizaje Cooperativo y Habilidades Sociales

\section{Interpretación:}

La tabla 1 muestra que existe predominio del nivel muy bueno de la variable aprendizaje cooperativo con 59,7\% (89 estudiantes), con menor predominio en los niveles bueno y regular con $38,9 \%$ (58 estudientes) y $1,3 \%$ ( 2 estudiantes). Como se observa, el mayor nivel está en el nivel muy bueno, ratificado por (Kagan, 2021) y (Tobón et al., 2010) el aprendizaje cooperativo estructurado asegurado ser una opción al aprendizaje tradicional por ser un método más flexible, eficaz, fácil y divertido, y para formar competencias en el aula es necesario promover éste tipo de aprendizaje, pasar del trabajo en grupo a la cooperación.

Tabla 2: Categorías de Habilidades Sociales

\begin{tabular}{llllll}
\hline & Frecuencia & Porcentaje & $\begin{array}{c}\text { Porcentaje } \\
\text { válido }\end{array}$ & $\begin{array}{c}\text { Porcentaje } \\
\text { acumulado }\end{array}$ \\
\hline Válido & REG & 1 &, 7 &, 7 &, 7 \\
BUE & 56 & 37,6 & 37,6 & 38,3 \\
MB & 92 & 61,7 & 61,7 & 100,0 \\
Total & 149 & 100,0 & 100,0 & \\
\hline
\end{tabular}

Nota. Data de Aprendizaje Cooperativo y Habilidades Sociales

\section{Interpretación:}

La tabla 2 muestra que existe predominio del nivel muy bueno de la variable habilidades sociales con 61,7\%, (92 estudiantes), con menor predominio en los niveles bueno y regular con $37,6 \%$ y $.7 \%$, (comprendido de 56 y 1 estudiantes respectivamente). Como 
se percibe, el mayor nivel está en el nivel muy bueno, corroborado por Kelly, (2002) cuando refiere que las habilidades sociales son comportamientos aprendidos que las personas utilizan en las relaciones humanas para resolver problemas con el fin de lograr sus metas.

Tabla 3: Prueba de Normalidad

\begin{tabular}{lccc}
\hline & \multicolumn{3}{c}{ Kolmogorov-Smirnov $^{\mathbf{a}}$} \\
\cline { 2 - 4 } & Estadístico & gl & Sig. \\
\hline INTERDEP &, 096 & 149 &, 002 \\
INTERV &, 093 & 149 &, 003 \\
RESPON &, 124 & 149 &, 000 \\
HABIL &, 122 & 149 &, 000 \\
EVAL &, 107 & 149 &, 000 \\
AC &, 052 & 149 &, $200^{*}$ \\
COND &, 083 & 149 &, 014 \\
COGN &, 104 & 149 &, 000 \\
SIT &, 096 & 149 &, 002 \\
HS &, 061 & 149 &, $200^{*}$ \\
\hline
\end{tabular}

Nota. Data de Aprendizaje Cooperativo y Habilidades Sociales

\section{Interpretación:}

De los resultados de la prueba de normalidad se puede apreciar que los valores obtenidos de sig. de aprendizaje cooperativo y habilidades sociales, son menores a 0.05 , precisandose que la distribución de los datos es No paramétrica; y por lo tanto, para la correlación de variables y dimensiones se debe utilizar la fórmula de Rho Spearman.

Tabla 4: Correlaciones del aprendizaje cooperativo y las habilidades sociales

\begin{tabular}{ll}
\hline \multicolumn{1}{c}{ Rho de Spearman } & HS \\
\hline AC Coeficiente de correlación &, $645^{* *}$ \\
Sig. (bilateral) &, 000 \\
$\mathrm{~N}$ & 149 \\
\hline
\end{tabular}

Nota. Data de Aprendizaje Cooperativo y Habilidades Sociales

\section{Interpretación:}

Se determina la existencia de una relación directa y altamente significativa entre el aprendizaje cooperativo y las habilidades sociales $\mathrm{r}=, 645^{* *}$ (correlación alta). Esta relación existente, converge con la idea de Estrada et al., (2016) cuando indica que debe existir una armonización entre el aprendizaje socializado sinérgico, incrementado en los 
discentes por una acción empírica y el manejo magistral de la afectividad y socialización de los estudiantes.

\section{DISCUSIÓN}

Como resultado global de la investigación se concluye que el aprendizaje cooperativo ha influído favorable y significativamente en el desarrollo de las habilidades sociales de los estudiantes de la Universidad César Vallejo de Trujillo 2020, cuya medida del coeficiente de correlación de Rho Spearman es de 0.645, reflejando una alta relación directa con nivel de significancia $0.01(\mathrm{p}<0.01)$, (Tabla17). En tal sentido, dichos resultados son corroborado por Mendo, (2019) en su investigación: "Desarrollo de habilidades sociales y de trabajo en equipo en el contexto universitario: Aprendizaje cooperativo y entrenamiento en habilidades sociales". Los resultado confirmaron que existe una correlación significativa entre las dos variables de estudio y que el uso de la metodología cooperativa en el contexto universitario brinda a los estudiantes la oportunidad de fomentar las capacidades relacionales, sociales y de colaboración que pueden ser decisivo en su éxito profesional y social.

Asimismo,se tiene que según Sánchez et al., (2019) en su investigación: Experiences of cooperative work in higher education. Perceptions about its contribution to the development of social competence, donde concluye que existe un vínculo muy positivo entre el trabajo cooperativo y el desarrollo de la competencia social relacionadas con la empatía, asertividad y habilidades relacionales en el ámbito personal y profesional en respuesta a las demandas y requerimientos de la sociedad y del mercado laboral. Esta investigación ratifica y fortalece nuestros resultados estadística y argumentativamente.

Igualmente, Mejía, (2019) en su estudio: Aprendizaje cooperativo y habilidades sociales en estudiantes de medicina de una universidad de Lima, quien parcialmente corrobora que existe relación estadística significativa $\mathrm{r}=0.585$ (correlación moderada) entre los dos componentes de los estudiantes de medicina de una universidad de Lima, con una significancia de $\mathrm{p}=0.000$, siendo ésta menor a 0.05 . Como se percibe dichos resultados son casi similares y coherentes con lo conseguido en la investigación

Por otro lado, los resultados obtenidos también son fortalecidos con la teoría de la interdependencia social, conocida también como, el aprendizaje cooperativo en el aula de los hermanos David y Roger Johnson, (1999) ha demostrado que el trabajo cooperativo, no solo enriquece el campo educativo del estudiante, sino también favorece 
la integración de todos los miembros del equipo, potenciando sus habilidades comunicativas y socio afectivas.

Igualmente, los resultados obtenidos también son fortalecidos por Pegalajar, (2018) en su trabajo de investigación: Formación en competencias en alumnado universitario de Educación Social mediante prácticas basadas en el aprendizaje cooperativo, donde concluye que existe una correlación buena y positiva $(\mathrm{M}=3.03 ; \mathrm{SD}=.49)$ entre el aprendizaje cooperativo y el desarrollo de las competencias referidas a las relaciones interpersonales, trabajo en equipo e interdisciplinar, compromiso ético, entre otros. Como se percibe dichos resultados tienen coherencia con lo obtenido en la investigación.

Otro aporte que refuerza dichos resultados es el de Huambachano y Huaire, (2018) en su investigación: Desarrollo de habilidades sociales en contextos universitarios, cuyo objetivo fue evaluar el efecto de un programa educativo en la mejora de las habilidades sociales en un grupo de estudiantes de la Universidad Nacional de Educación Enrique Guzmán y Valle. Los resultados confirman que, a través de metodologías activas y materiales apropiados, trabajar para mejorar las habilidades sociales en un determinado momento y contexto, a través de un programa educativo, mejora las prácticas prosociales y la conducta de los alumnos.

Otro hallazgo que parcialmente concuerda, son los conseguidos por León et al., (2015) en su estudio de investigación: Habilidades sociales en equipos de aprendizaje cooperativo en el contexto universitario, donde ratifica que existe una correlación significativa entre ambas variables. La intención del aprendizaje en grupo es fortalecer alos miembros académica y emocionalmente.Trabajar en equipos a nivel universitario asumiendo responsabilidad a nivel personal y grupal como estrategia metodológica del aprendizaje cooperativo, contribuye en las habilidades sociales del equipo y éstas son básicas para ser eficaz en otras situaciones y escenarios cotidianos de trabajo cooperativo

Igualmente, los resultados obtenidos también son fortalecidos, en la medida que la teoría de las habilidades sociales, inspirado en la teoria de las "inteligencias múltiples" de Gardner, (2019), nos dice que la inteligencia interpersonal es la capacidad de comprender a los demás y como tal, es de vital importancia dentro de de los equipos de trabajo cooperativo, que a la vez involucra otros factores como: habilidad de liderazgo, 
capacidad para resolver conflictos y un análisis social adecuado. De manera análoga esto también sucede en los estudiantes de la universidad César Vallejo de Trujillo, donde se identificó un alto nivel de relación entre ambas variables de estudio. En esta misma perspectiva tenemos la teoria de la "inteligencia emocional" de Goleman, (2005) que incluye dos tipos de inteligencia: la intrapesrsonal y la interpersonal. El primero tiene que ver con la autoestima y el segudo tiene que ver con las habilidades socilaes, como la capacidad de gestionar nuestras emociones y las de los demás.

\section{CONCLUSIÓN}

Se concluyó que, el aprendizaje coopeartivo ha influído favorable y significativamente en el desarrollo de las habilidades sociales de los estudiantes de la universidad César Vallejo de Trujillo 2020, cuya medida del coeficiente de correlación Rho Spearman es $\mathrm{r}=, 645^{* *}($ correlación alta $) ; \mathrm{p}<0.01$

\section{RECOMENDACIONES}

Las recomendaciones realizadas en este estudio tienen como objetivo mostrar los aspectos positivos como las dificultades que se encontraron en el transcurso y desarrollo de dicho estudio, a fin que directivos, educadores e investigadores, en una futura investigación los consideren y cooperen en favor de la calidad educativa.

- La recomendación principal está dirigida no solo a los directivos de la Universidad César Vallejo, sino también a los vicerrectores académicos y de investigación de todas las instituciones superiores de formación profesional, la que consiste en planificar y desarrollar un programa de actualización docente respecto a estas dos variables de estudio que son el aprendizaje cooperativo y las habilidades sociales, con el objetivo de poner mayor interes, atención y cuidado en las características y peculiaridades individuales de los estudiantes, a fin de saber reconocer y diferenciar sus potencialidades y carencias en pro de obtener una mejor formación universitaria. Todo esto se evidencia, que un $49 \%$ de la dimensión de la interdependencia positiva no está en el nivel muy bueno para mejorar las habilidades sociales. De igual manera, debe hacerse con la intervención promocional cara a cara en el que 53,7\% no está en el nivel muy bueno. Así mismo, se debe proceder con la responsabilidad individual y grupal que está con $45 \%$ en el nivel no muy bueno para elevar las habilidades sociales. De igual modo, respecto a la dimensión de las habilidades interpersonales y grupales el 38,3\% no está en el nivel muy bueno para incrementar las habilidades 
sociales. Por último, la evaluación grupal con 54,4\% no está en el nivel muy bueno para acrecentar y desarrollar las habilidades sociales de los estudiantes de la Universidad César Vallejo de Trujillo.

- Los docentes universitarios deben poner mayor atención a las características de sus estudiantes, tomando en cuenta que los efectos positivos del trabajo cooperativo frente a una metodología competitiva e individualista ha dado mejor resultados. Entendiendo que a mayor compromiso de los integrantes del equipo, mayor resulta la implicación en la tarea a realizar. Así mismo, los estudintes descubren que esta metodología de aprendizaje les permitió desarrollar habilidades sociales y que esta habilidad está unida a la resolución de problemas y conflictos interpersonales.

- A los investigadores, profundizar y hacer una investigación más exhaustiva sobre las mismas variables de estudio, a fin de obtener y optimizar dichos resultados, por lo mismo, que en el Perú existen escasas investigaciones en el contexto universitario.

\section{REFERENCIAS BIBLIOGRÁFICAS}

Antón, M. (2010). Aportaciones de la teoría sociocultural al estudio de la adquisición del español como segunda lengua. Revista española de lingüística aplicada, 23, 9-30.

Baquero Torres, M. I., \& Rendón Acevedo, J. A. (2011). Desarrollo humano local: la alternatividad para el buen vivir. Revista Universidad de La Salle, 54, 67-86. https://ciencia.lasalle.edu.co/cgi/viewcontent.cgi?article=1047\&context=ruls

Caballo, V. (2007). Manual de evaluación y entrenamiento-de las habilidades sociales (Editores Siglo XXI de España (ed.); 7. ${ }^{\mathrm{a}}$ ed.). https://cideps.com/wpcontent/uploads/2015/04/Caballo-V.-Manual-de-evaluación-y-entrenamiento-delas-habilidades-sociales-ebook.pdf

Cervantes, L., Leyva, B., Rivas, P., \& Zavala, C. (2018). Relación profesor - alumno para fomentar el aprendizaje cooperativo de los estudiantes del $\mathrm{V}$ ciclo de la USAT. EDUCARE ET COMUNICARE: Revista de investigación de la Facultad de Humanidades, 2(2), 90-98. http://revistas.usat.edu.pe/index.php/educare/article/view/121/468

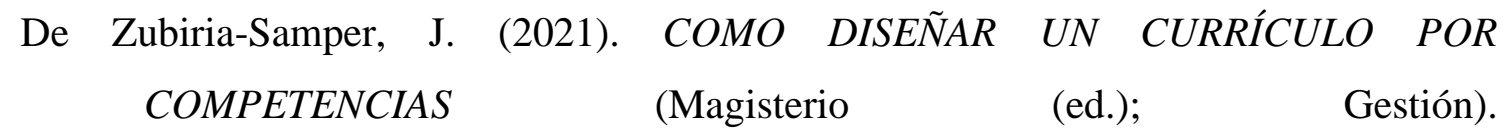
http://bibliotecadigital.magisterio.co/libro/como-dise-ar-un-curr-culo-por- 
competencias

Estrada, M., Monferrer, D., \& Moliner, M. A. (2016). Cooperative Learning and SocioEmotional Skills: A Teaching Experience in Sales Techniques Course. Formación universitaria, 9(6), 43-62. https://dx.doi.org/10.4067/S071850062016000600005

Gardner, H. (2019). Inteligencias múltiples. La teoría en la práctica. (Editorial planeta S.A. (ed.)). Paidos Educación. https://www.planetadelibros.com/libros_contenido_extra/42/41371_INTELIGE NCIAS_MULTIPLES.pdf

Goleman,

D. (2005).

Inteligencia

Emocional

(Kairós).

http://www.codajic.org/sites/www.codajic.org/files/Inteligencia

Emocional Daniel Goleman.pdf

Hernández-sampieri, R., Fernández-Collao, C., \& Baptista-Lucio, P. (2014). METODOLOGÍA DE LA INVESTIGACIÓN. (Mc Graw Hill Education (ed.); Sexta edic). McGRAW-HILL / INTERAMERICANA EDITORES, S.A. DE C.V. https://www.uca.ac.cr/wp-content/uploads/2017/10/Investigacion.pdf

Hernández-sampieri, R., \& Mendoza-Torres, C. (2018). Metodología de la investigación - Las rutas cuantitativa, cualitativa y mixta. En McGRAW-HILL INTERAMERICANA EDITORES S.A. de C. V (Ed.), Mac graw hill education.

Huambachano, A. M., \& Huaire, E. (2018). Desarrollo de habilidades sociales en contextos universitarios. Horizonte de la Ciencia, 8(14), 123-130. http://revistas.uncp.edu.pe/index.php/horizontedelaciencia/article/view/300/317

Johnson, D. W., Johnson, R. T., \& Holubec, E. J. (1999). El aprendizaje cooperativo en el aula. ResearchGate, $\quad 4(3), 12$. https://www.researchgate.net/publication/265567256

Johnson, David W., \& Johnson, R. T. (1999). Aprender Juntos y Solos. Grupo Editorial Aique S. A. https://santander.edu.mx/aula/pluginfile.php/705/mod_resource/content/2/Apren der juntos y solos_David W. Jonson y Roger J. Jonson.pdf

Kagan, S. (2021). Kagan Structures and Learning Together: What is the Difference? Revista Kagan, $1-9$. https://www.kaganonline.com/free_articles/dr_spencer_kagan/275/Kagan- 
\%09Structures-and-Learning-Together-What-is-the-Difference

Kelly, J. (2002). Entrenamiento de las habilidades sociales (Desclée De Brouwer (ed.); 8. ${ }^{\text {a }} \quad$ ed., Vol. 53, Número 9). Biblioteca de Psicología. https://www.edesclee.com/img/cms/pdfs/9788433007025.pdf

León, B., Felipe, E., Mendo, S., \& Iglesias, D. (2015). Habilidades sociales en equipos de aprendizaje cooperativo en el contexto universitario. Behavioral Psychology/ Psicologia Conductual, 23(2), 191-214. https://www.researchgate.net/publication/285198652_Habilidades_sociales_en_ equipos_de_aprendizaje_cooperativo_en_el_contexto_universitario

Mejía-Castillo, C. (2019). Aprendizaje cooperativo y habilidades sociales en estudiantes de medicina de una universidad de Lima, 2019. En Repositorio Institucional UCV. https://hdl.handle.net/20.500.12692/38628

Mendo-Lázaro, S. (2019). «Desarrollo de Habilidades Sociales y de Trabajo en Equipo en el contexto universitario: Aprendizaje Cooperativo y Entrenamiento en Habilidades Sociales». Tesis Doctoral [Universidad de Extremadura.]. http://dehesa.unex.es/handle/10662/9276

Pegalajar Palomino, M. del C. (2018). Formación en competencias en alumnado universitario de Educación Social mediante prácticas basadas en el aprendizaje cooperativo. Revista Complutense de Educacion, 29(3), 829-845. https://doi.org/10.5209/RCED.53970

Rodríguez, L., Cacheiro, M., \& Gil, J. (2014). Desarrollo de habilidades sociales en estudiantes mexicanos de preparatoria a través de actividades virtuales en la plataforma Moodle. Education in the Knowledge Society (EKS), 15(3), 149-171. https://revistas.usal.es/index.php/eks/article/view/12222/12567

Sanchez- Marin, F. J., Parra-Merono, M. C., \& Pena-Acuna, B. (2019). Experiences of Cooperative Work in Higher Education. Perceptions About Its Contribution To the Development of Social Competence. Vivat Academia, 147, 87-107. https://doi.org/https://doi.org/10.15178/va.2019.147.87-108

Sánchez, H., Reyes, C., \& Mejía, K. (2018). Manual de términos en investigación científica, tecnológica y humanística (Bussiness Support Aneth S.R.L. (ed.)). Universidad Ricardo Palma. https://www.urp.edu.pe/pdf/id/13350/n/libromanual-de-terminos-en-investigacion.pdf 
Sharan, Y. (2014). Learning to cooperate for cooperative learning. [Aprendiendo a cooperar en el aprendizaje cooperativo]. Anales de Psicología, 30(3), 802-807. https://doi.org/10.6018/analesps.30.3.201211

Tobón, S., Pimienta, J., \& García, J. (2010). Secuencias didácticas: aprendizaje y evaluación de competencias. Pearson Educación de México, S.A. de C.V. http://files.ctezona141.webnode.mx/200000004-8ed038fca3/secuenciasdidacticastobon-120521222400-phpapp02.pdf 